\title{
Identification of potential biomarkers for differential diagnosis between rheumatoid arthritis and osteoarthritis via integrative genome-wide gene expression profiling analysis
}

\author{
RONGQIANG ZHANG ${ }^{1,2}$, XIAOLI YANG ${ }^{1}$, JING WANG ${ }^{2}$, LIXIN HAN $^{1}$, AIMIN YANG $^{3}$, \\ JIE ZHANG ${ }^{3}$, DANDAN ZHANG ${ }^{1}$, BAORONG LI ${ }^{1}$, ZHAOFANG LI $^{1}$ and YONGMIN XIONG ${ }^{1}$ \\ ${ }^{1}$ School of Public Health, Xi'an Jiaotong University Health Science Center, Key Laboratory of Trace Elements \\ and Endemic Diseases of The National Health and Family Planning Commission, Xi'an, Shaanxi 710061; \\ ${ }^{2}$ School of Public Health, Shaanxi University of Chinese Medicine, Xianyang, Shaanxi 712046, P.R. China; \\ ${ }^{3}$ School of Public Health, Brown University, Providence, RI 02906, USA
}

Received May 31, 2018; Accepted September 24, 2018

DOI: $10.3892 / \mathrm{mmr} .2018 .9677$

\begin{abstract}
The present study aimed to identify potential novel biomarkers in synovial tissue obtained from patients with Rheumatoid Arthritis (RA) and Osteoarthritis (OA) for differential diagnosis. The genome-wide expression profiling datasets of synovial tissues from RA and OA cohorts, including GSE55235, GSE55457 and GSE55584 datasets, were retrieved and used to identify differentially expressed genes (DEGs; $\mathrm{P}<0.05$; false discovery rate $<0.05$ and Fold Change >2) between RA and OA using R software. Gene Ontology and Kyoto Encyclopedia of Genes and Genomes pathway enrichment analyses of DEGs were performed to determine molecular and biochemical pathways associated with the identified DEGs, and a protein-protein interaction (PPI) network of the DEGs was constructed using Cytoscape software. Significant modules in the PPI network and candidate driver genes were screened using the Molecular Complex Detection Algorithm. Potential biomarkers were evaluated by receiver operating characteristic and logistic regression analyses. Large numbers of DEGs were detected, including 273, 205 and 179 DEGs in the GSE55235, GSE55457 and GSE55584 datasets, respectively. Among them, $80 \mathrm{DEGs}$ exhibited identical expression trends in all the three datasets, including 49 upregulated and 31 downregulated genes in patients with RA. DEGs in patients suffering from RA compared with patients suffering from OA were predominantly associated with the primary
\end{abstract}

Correspondence to: Professor Yongmin Xiong, School of Public Health, Xi'an Jiaotong University Health Science Center, Key Laboratory of Trace Elements and Endemic Diseases of The National Health and Family Planning Commission, 76 Yanta West Road, Xi'an, Shaanxi 710061, P.R. China

E-mail: xiongym@mail.xjtu.edu.cn

Key words: rheumatoid arthritis, osteoarthritis, pathway, protein-protein interaction, sensitivity, specificity immunodeficiency pathway, including interleukin 7 receptor (IL7R) and signal transducer activator of transcription 1 (STAT1). The sensitivity of IL7R + STAT1 to differentiate RA from OA was $93.94 \%$ with a specificity of $80.77 \%$. The results generated from analyses of the GSE36700 dataset were closely associated with results generated from analyses of GSE55235, GSE55457 and GSE55584 datasets, which further verified the reliability of the aforementioned results. The results of the present study suggested that increased expression of $I L 7 R$ and STAT1 in synovial tissue as well as in the primary immunodeficiency may be associated with RA occurrence. These identified novel biomarkers may be used to predict disease occurrence and clinically differentiate RA from OA.

\section{Introduction}

Rheumatoid arthritis (RA) and osteoarthritis (OA) are the two most frequent types of degenerative joint diseases and exhibit similar etiology $(1,2)$. RA is a complex, chronic inflammatory and autoimmune arthritis that typically causes pain, swelling, stiffness and loss of function in the joints (1). It has been estimated that RA affects $0.5-1 \%$ of the adult population worldwide with 20-50 novel cases per 100,000 people occurring annually, which most frequently occurs in women aged $>40$ years old $(3,4)$. RA has become one of the most common causes of reduced productivity and disability in affected patients and may additionally pose a substantial financial burden on the family of the patient as well as society (5). RA manifests as osteoporosis around the joint and joint space narrowing in the knees of patients (6). The bone anatomy degeneration and cystic degeneration of the bone joint surface may additionally occur with bone defects (7). During RA, the intercondylar fossa is enlarged and the tibial plateau sinks $(8,9)$. Patients with late-stage RA may suffer from articular surface sclerosis, joint subluxation or joint stiffness (10). Furthermore, OA, the most prevalent form of arthritis worldwide, is a multi-gene and multi-factorial disease, and is characterized by cartilage degeneration and subchondral bone alterations, involving synovial tissue and articular cartilage (10-12). OA may reduce 
the quality of life for patients and eventually lead to disability due to pain. The joint most commonly affected by OA is the knee (8). Similar to RA, OA additionally has an increased occurrence rate in older adults, particularly in women.

In routine clinical practice, the diagnostic criteria for RA and OA are outlined by the American College of Rheumatology (Atlanta, USA). RA and OA exhibit overlapping symptoms, making differential diagnosis particularly challenging. In addition, differentiation between RA and $\mathrm{OA}$ is difficult in late-stage cases, primarily because disease progression frequently begins prior to the onset of symptoms. Therefore, accurate diagnosis of RA and OA may significantly improve the clinical outcomes and prognosis for affected patients. However, the mechanisms underlying the initiation and progression of RA and OA remain unclear. Previously, important genes and diagnostic markers that interact with each other and with environmental and stochastic factors have been identified in the two diseases (13). However, these markers may not entirely elucidate the complex pathogenesis of RA and OA.

Therefore, the present study aimed to investigate the developmental differences between RA and OA. An updated comprehensive analysis was performed to identify the potential novel biomarkers associated with synovial tissues obtained from patients with RA and OA. In the present study, three multicenter genome-wide transcriptomic datasets, including 33 patients with RA and 26 patients with OA were retrieved and analyzed. The present study aimed to investigate the different mechanisms underlying the differential pathogenesis of RA and OA, and thus improve the diagnosis and treatment strategies available for patients suffering from the two diseases in clinical practice.

\section{Materials and methods}

Microarray dataset source. A systematic search of microarray datasets was performed to examine differentially expressed genes (DEGs) between RA and OA. The National Center for Biotechnology Information's Gene Expression Omnibus (GEO) database (http://www.ncbi.nlm.nih.gov/geo/) was utilized to retrieve appropriate microarray datasets. The key words 'Osteoarthritis' and 'Rheumatoid Arthritis' were used for the screening. Datasets were included if they met the following inclusion criteria: i) were based on gene expression profiling of synovial membrane samples from the same platform. When the microarray datasets are obtained from the same platform, their homogeneity is usually good. Subsequent to screening OA-associated microarray datasets, the GPL96 platform was used at the highest frequency. Therefore, the OA-associated microarray datasets obtained from the GPL96 platform were included in the present study; ii) case (RA)-control (OA) studies; iii) patients with RA were diagnosed and classified based on the American College of Rheumatology criteria (14) and patients with OA were classified according to the criteria of Diagnostic and Therapeutic Criteria Committee of the American Rheumatism Association (15); and iv) the number of synovial tissue samples in each group of patients with RA and OA was $\geq 6$. Three gene expression datasets, GSE55235 $\left(\mathrm{n}_{\mathrm{RA}}=10\right.$ and $\left.\mathrm{n}_{\mathrm{OA}}=10\right)$, GSE55584 $\left(\mathrm{n}_{\mathrm{RA}}=10\right.$ and $\left.\mathrm{n}_{\mathrm{OA}}=6\right)$ and GSE55457 $\left(\mathrm{n}_{\mathrm{RA}}=13\right.$ and $\left.\mathrm{n}_{\mathrm{OA}}=10\right)$ met the inclusion criteria and were included in the present study (16).
A further dependent dataset, GSE36700 (https://www. ncbi.nlm.nih.gov/geo/query/acc.cgi?acc=GSE36700) (17), including microarray data from synovial biopsies of patients with RA (n=7) and OA ( $=5)$, was used to validate the results obtained from the GSE55235, GSE55584 and GSE55457 datasets. The GSE36700 dataset was created based on the Affymetrix Human Genome U133 Plus 2.0 Array (Affymetrix UK Ltd., High Wycombe, UK) and was submitted by Nzeusseu Toukap et al (17) March 22, 2012 and updated on Aug 09, 2018. Patients with RA were diagnosed and classified based on the 1987 American College of Rheumatology criteria (14) and patients with OA were classified according to X-ray evidence of osteoarthritis (15).

Data preprocessing and differential expression analysis. The three databases were created based on the Affymetrix Human Genome U133A Array (Affymetrix UK Ltd.). A robust multi-array average algorithm using the Affy package (justMRA; http://ugrad.stat.ubc. ca/R/library/affy/html/00Index.html) was conducted for background adjustment, normalization and summarization of the three datasets to minimize data inconsistency and heterogeneity. The probe sets were converted into corresponding gene annotation using $\mathrm{R} / \mathrm{Bioconductor}$ package (version 3.22.4; http://www.bioconductor.org/packages/release/BiocViews.html\#___ChipManufacturer) and the Affymetrix Human Genome U133 Plus 2.0 Array. The probes with no gene annotation were excluded from the analysis. The expression values of all probes for a given gene were calculated from the average expression value. DEGs $[\mathrm{P}<0.05$; false discovery rate $(\mathrm{FDR})<0.05$ ] between RA and OA from the three datasets were investigated using $\mathrm{R}$ software (v3.4.0; http://bioconductor.org/biocLite.R). FDR was applied based on the Benjamini \& Hochberg method (18) and two independent sample Student's t-test was performed to select sets of DEGs. To reduce the false positive rate, DEGs of the three datasets were identified, and subsequently Venn diagrams (Venn 2.1; http://bioinfogp.cnb.csic.es/tools/venny/index. html) were used to screen the overlapping DEGs of the three datasets to improve the stability of the subsequent results.

Biological functions and pathway enrichment analyses. To further elucidate the biological functions of DEGs between RA and OA, the identified DEGs were subjected to Gene Ontology (GO; http://www.geneontology.org/) term enrichment and Kyoto Encyclopedia of Genes and Genomes (KEGG; https://www.kegg.jp/) pathway analyses using Database for Annotation, Visualization and Integrated Discovery (DAVID; https://david.ncifcrf.gov/), respectively. Significantly enriched pathways and GO terms $(\mathrm{P} \leq 0.05$; number of enrichment genes $\geq 2$ ) were identified using Cytoscape 3.5.1 software (http://www.softpedia. com/get/Science-CAD/Cytoscape.shtml) and GeneClip 2.0 (http://gsds.cbi.pku.edu.cn/). The identified genes were classified into three functional categories, including the Biological Process (BP), Molecular Function (MF) and Cellular Component (CC). Fisher's exact tests (two-sided) or $\chi^{2}$ tests were performed to categorize the pathway and GO terms. The FDR (Benjamini \& Hochberg method) (18) was applied to obtain the corrected P-values. Significantly 
enriched pathways and GO terms (P£0.05; number of enrichment genes $\geq 2$ ) were identified using Cytoscape 3.5.1 software.

Analysis of pathway networks. The establishment of a pathway network of DEGs between RA and OA may help to identify important pathways associated with the development of RA and OA. Furthermore, pathway network analysis may reveal the possible interactions and crosstalk among these pathways. Therefore, pathway networks were constructed based on the identified DEGs using the ClueGO plugin on the Cytoscape platform (http://www.softpedia. com/get/Science-CAD/Cytoscape.shtml).

Protein-protein interaction (PPI) network analysis of DEGs. The Search Tool for the Retrieval of Interacting Genes/Proteins (STRING) database (http://string-db.org/) was used to analyze the PPI of DEGs, and Cytoscape software was used to construct the PPI network. FDR $<0.05$ was considered as the cut-off criterion. A network was constructed consisting of nodes and lines in which each node represents a protein and the lines represent direct interactions between proteins. The PPI network was constructed based on human data alone. The number of nodes that may interact with a given node was expressed as the degree of the node. The greater the degree values of the included genes, the greater the degree in the whole network.

Identification of candidate genes between RA and $O A$. Identification of genes that may affect the development of $\mathrm{RA}$ and $\mathrm{OA}$ within the genome may provide a comprehensive understanding of the differences between the pathogenesis of RA and OA. Candidate genes that may affect the development of RA and OA based on the DEGs were predicted using Molecular Complex Detection Algorithm (MCODE) in Cytoscape (19). Furthermore, MCODE cluster analysis was performed using Cytocluster 3.5.1 software (20) (Degree cutoff $=2$; Node score cutoff $=0.2 ; \mathrm{K}$-core $=2$; Max Depth=100) to identify the most significant MCODE clusters, according to clustering scores. The GeneMANIA Cytoscape plugin was used to identify and prioritize novel candidate genes involved in RA and OA. The establishment of entire PPI networks of DEGs between RA and OA were identified based on the biological network using the GeneMANIA plugin (21). The PPI networks were composed of genes included in the list of 80 DEGs and predicted candidate genes that may affect the development of RA and OA. Following the selection of Homo sapiens as the organism, common DEGs were entered into the GeneMANIA search bar, and the PPI network was constructed. Following this, the whole PPI network was filtered using a degree-filtering approach to include the most critical biomarkers in the occurrence of the two diseases using Cytoscape 3.5.1 software.

Statistical analysis. The raw expression data of patients with RA and OA were obtained from GEO datasets and logarithmically transformed. The means of two continuous, normally distributed variables were compared by independent sample Student's t-tests. Mann-Whitney U tests were used to compare the means of two groups of variables not normally distributed. Receiver Operating Characteristic (ROC) analysis was performed to identify a more accurate cut-off point in the gene expression level, which may aid the classification of RA and OA. ROC curves were generated by plotting the range of sensitivity (true positive fractions) and specificity (false positive fractions) pairs for each subject's error rate, with case status (RA vs. OA) representing the classifier variable. Youden's index was used for capturing the performance of a dichotomous diagnostic test. Youden's index=sensitivity + specificity -1 (22).

The area under the ROC curve (AUC), which provides an estimate of the accuracy of the diagnostic test for the discrimination between patients with RA and patients with $\mathrm{OA}$, was used to assess the performance of the test. Binary logistic regression using backward stepwise selection mode was performed to screen out potential biomarkers that were positively correlated with RA diagnosis when identified biomarkers were detected together. Following this, ROC analysis was performed to determine the performance of the established logistic regression models. All statistical analyses in the present study (except for the screening of DEGs) were performed using SPSS version 24.0 for Windows (IBM Corp., Armonk, NY, USA) and GraphPad Prism 7.0 (GraphPad Software, Inc., La Jolla, CA, USA). P $<0.05$ was considered to indicate a statistically significant difference.

\section{Results}

Identification of DEGs between patients with RA and OA. DEGs were identified by the t-test statistical algorithm. Based on the cutoff criteria, 140, 103 and 95 genes were identified in GSE55235, GSE55457 and GSE55584 datasets, respectively, which were upregulated in patients with RA (Fig. 1A-C). In addition, 133, 102 and 84 genes were identified in GSE55235, GSE55457 and GSE55584 datasets, respectively, which were downregulated in patients with RA (Fig. 1A-C). Notably, 50 upregulated (Fig. 1D) and 31 downregulated DEGs (Fig. 1E) in patients with RA were identified as being overlapped between the three datasets. One DEG without a symbol was excluded from the upregulated DEGs, therefore 80 DEGs in total, containing 49 upregulated and 31 downregulated, were included in the final analysis. The list of 80 DEG symbols is available upon request.

Biological functions and KEGG pathways. Cytoscape 3.5.1 and GeneClip 2.0 were used for biological function and pathway enrichment analyses. The results of these analyses identified 80 overlapped DEGs, which are presented in Table I. The results demonstrated that 80 overlapped DEGs were significantly enriched in immune, inflammation, apoptosis and antioxidant stress-associated functions and pathways.

PPI network analysis. A PPI network was constructed based on the biological interactions of the 80 identified DEGs to further elucidate their associations at the protein level. As presented in Fig. 2A, 32 nodes were screened out, including 29 upregulated genes and three downregulated genes (heparin-binding epidermal growth factor-like growth factor, ephrin type-A receptor 3 and clusterin) in patients with RA. The PPI network included three primary sub-clusters: i) A 

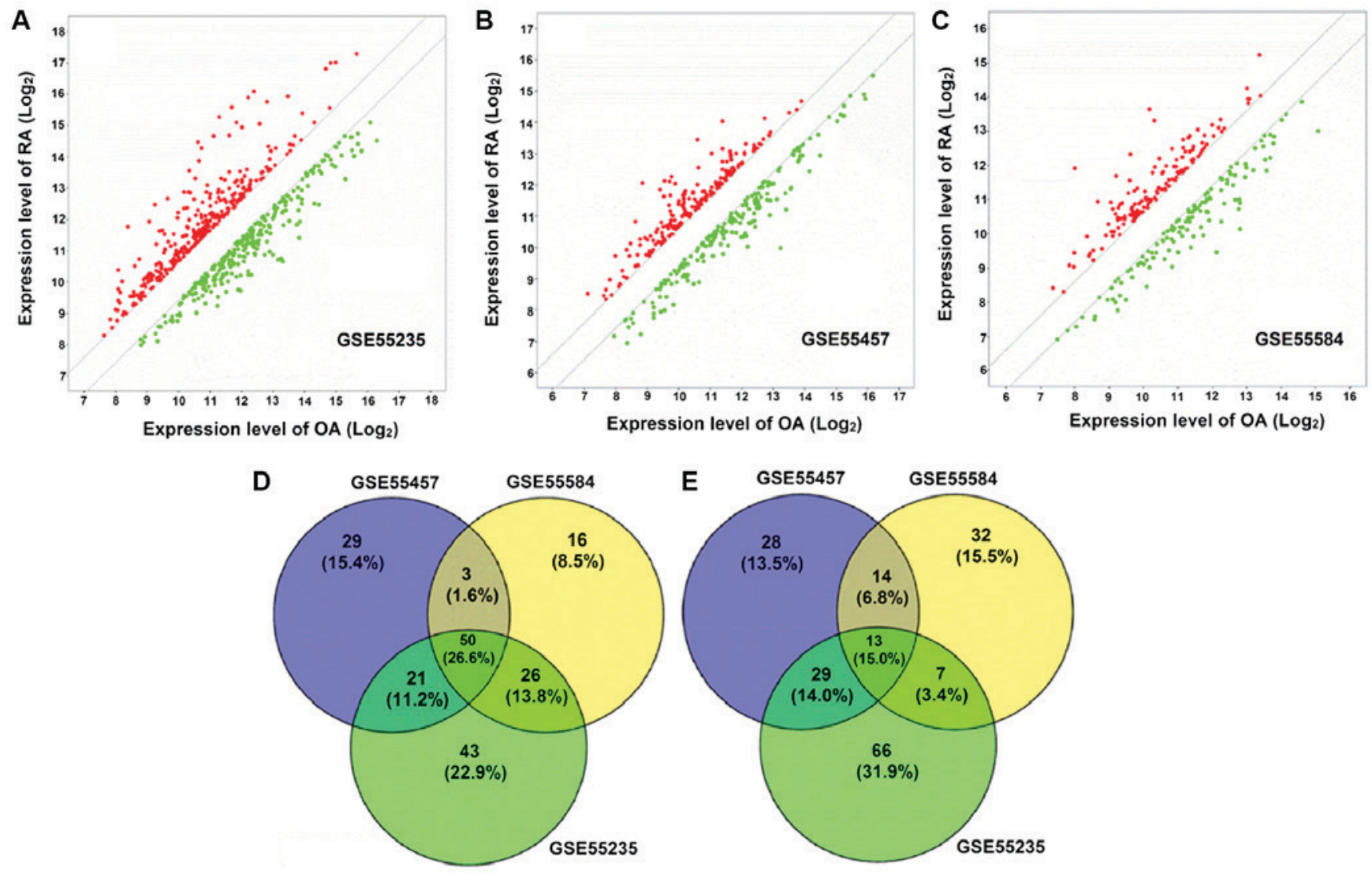

Figure 1. Identification of DEGs between patients with RA and OA. (A) In total, 140 upregulated and 133 downregulated genes were identified in patients with RA from GSE55235 datasets. (B) In total, 103 upregulated and 102 downregulated genes were identified in patients with RA from GSE55457 datasets. (C) In total, 95 upregulated and 84 downregulated genes were identified in patients with RA from GSE55584 datasets. (D) In total, 50 upregulated DEGs in patients with RA were identified as being overlapped between the three datasets. (E) In total, 31 downregulated DEGs in patients with RA were identified as being overlapped between the three datasets. One DEG without a symbol was excluded from the upregulated DEGs, therefore 80 DEGs in total were included in the final analysis. RA, rheumatoid arthritis; OA, osteoarthritis; DEG, differentially expressed gene.

sub-cluster including $C$-X-C motif chemokine receptor 4 (CXCR4) and signal transducer and activator of transcription 1 (STAT1), and at its core was predominantly associated with chemokines and immune functions (Fig. 2A; top circle); ii) a sub-cluster including $L C K$ proto-oncogene, Src family tyrosine kinase $(L C K)$, and at its core was predominantly correlated with the regulation of developmental events, notably in the nervous system (Fig. 2A; middle circle); iii) a sub-cluster including interleukin (IL)2 receptor, $\gamma$ chain (IL2RG) and CD3d molecule (CD3D), and at its core was primarily associated with immunodeficiency (Fig. 2A; bottom circle).

Candidate genes and core network. To identify the potential pathological molecular network of the 80 identified DEGs, the specific network among them based on the human interactome network using MCODE algorithm was extracted using the default settings on GeneMANIA. This approach included maximal members of candidate genes with the minimal interaction associations. The network, capturing the 80 DEGs as its seeds, contained 323 nodes and 602 edges, including 30 genes of the 80 DEGs and 293 candidate genes that may affect RA and OA progression. To identify the most important core network, networks were filtered according to their degree using the degree-filtering approach. Finally, a core network including six genes (CD3D, CXCR4, IL2RG, IL7R, LCK and STATI) was identified and presented in Fig. 2B, which suggested that these six genes may represent important biomarkers associated with RA and OA development and diagnosis.

Gene-pathway network. To further understand how important genes in the core PPI network affect RA development, a gene-pathway network was constructed using ClueGO (Fig. 2C). From the gene-pathway network, five genes $(C D 3 D, I L 2 R G$, $I L 7 R, L C K$ and STAT1) were included in the PPI network. Notably, the results demonstrated that $C D 3 D, I L 2 R G, I L 7 R$, $L C K$ and STAT1 interacted with the primary immunodeficiency pathway, either directly or indirectly $(\mathrm{P}<0.001)$, which suggested that upregulated genes may activate the primary immunodeficiency pathway and increase the risk of RA development.

Evaluation of the core network for RA identification. ROC curves were constructed to calculate the AUCs of $C D 3 D$ (0.8357), CXCR4 (0.7855), IL2RG (0.8368), IL7R (0.9161), LCK (0.8683) and STAT1 (0.9138; all $\mathrm{P}<0.0001)$. Taking the maximum value of the Youden's index, the $\log _{2}$ expression value of $C D 3 D$ (8.65), CXCR4 (10.86), IL2RG (9.27), IL7R (8.11), LCK (6.58) and STAT1 (7.36) were determined (Fig. 3A-F and Table II). For RA identification, at the ROC-derived optimum cut-offs, the highest sensitivity exhibited by STATl was $90.91 \%$. The 
Table I. Top five biological functions and top ten KEGG pathways of the overlapped differentially expressed genes.

A, Biological process

\begin{tabular}{llll}
\hline ID & \multicolumn{1}{c}{ GO term description } & Count & FDR \\
\hline GO:0007166 & Cell surface receptor signaling pathway & 38 & $1.10 \times 10^{-13}$ \\
GO:0051239 & Regulation of multicellular organismal process & 40 & $1.10 \times 10^{-13}$ \\
GO:0030155 & Regulation of cell adhesion & 23 & $1.23 \times 10^{-13}$ \\
GO:2000026 & Regulation of multicellular organismal development & 32 & $1.41 \times 10^{-12}$ \\
GO:0070887 & Cellular response to chemical stimulus & 37 & $6.78 \times 10^{-12}$ \\
\hline
\end{tabular}

B, Molecular function

\begin{tabular}{llrr}
\hline ID & \multicolumn{1}{c}{ GO term description } & Count & FDR \\
\hline GO:0005515 & Protein binding & 51 & $3.83 \times 10^{-11}$ \\
GO:0005102 & Receptor binding & 25 & $1.05 \times 10^{-09}$ \\
GO:0005003 & Ephrin receptor activity & 6 & $4.91 \times 10^{-08}$ \\
GO:0008201 & Heparin binding & 10 & $5.03 \times 10^{-07}$ \\
GO:0042802 & Identical protein binding & 20 & $5.17 \times 10^{-07}$ \\
\hline
\end{tabular}

C, Cellular component

\begin{tabular}{llll}
\hline ID & \multicolumn{1}{c}{ GO term description } & Count & FDR \\
\hline GO:0005615 & Extracellular space & 28 & $4.35 \times 10^{-11}$ \\
GO:0009986 & Cell surface & 19 & $2.35 \times 10^{-08}$ \\
GO:0005576 & Extracellular region & 42 & $8.80 \times 10^{-07}$ \\
GO:0009897 & External side of plasma membrane & 11 & $8.80 \times 10^{-07}$ \\
GO:0098552 & Side of membrane & 13 & $2.42 \times 10^{-06}$ \\
\hline
\end{tabular}

D, KEGG pathway

\begin{tabular}{llrr}
\hline ID & \multicolumn{1}{c}{ GO term description } & Count & FDR \\
\hline 4,060 & Cytokine-cytokine receptor interaction & 16 & $3.66 \times 10^{-12}$ \\
4,360 & Axon guidance & 9 & $3.42 \times 10^{-07}$ \\
4,062 & Chemokine signaling pathway & 10 & $3.69 \times 10^{-07}$ \\
5,340 & Primary immunodeficiency & 6 & $4.88 \times 10^{-07}$ \\
4,151 & PI3K/AKT signaling pathway & 7 & $8.41 \times 10^{-05}$ \\
4,630 & JAK-STAT signaling pathway & 6 & $1.87 \times 10^{-04}$ \\
5,162 & Measles & 5 & $7.14 \times 10^{-04}$ \\
4,640 & Hematopoietic cell lineage & 5 & $1.05 \times 10^{-03}$ \\
4,064 & NF- $\kappa$ B signaling pathway & 5 & $1.11 \times 10^{-03}$ \\
4,660 & T cell receptor signaling pathway & 5 & $1.52 \times 10^{-03}$ \\
5,142 & American trypanosomiasis & $52 \times 10^{-03}$ &
\end{tabular}

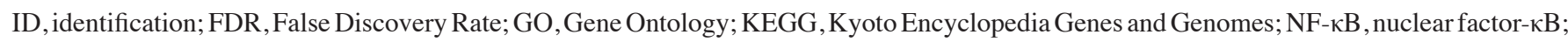
JAK, Janus kinase; STAT, signal transducer and activator of transcription; AKT, protein kinase B; PI3K, phosphatidylinositol-4,5-bisphosphate 3-kinase.

specificities exhibited by $C X C R 4, I L 2 R G$ and $I L 7 R$ were equal and reached a maximum value of $92.31 \%$.

Subsequent binary logistic regression analysis demonstrated that $I L 7 R$ [odds ratio $(\mathrm{OR})_{\text {RA vs. OA }}=4.551$; OR $95 \%$ confidence interval (CI): 1.517-13.657; $\mathrm{P}=0.007]$ and STAT1 $\left(\mathrm{OR}_{\mathrm{RA} \text { vs. OA }}=2.923\right.$; OR 95\% CI: 1.091-7.829; $\left.\mathrm{P}=0.033\right)$ were inputted into the regression, which suggested that $I L 7 R$ and STAT1 may be detected together (Fig. 3G and Table III). 

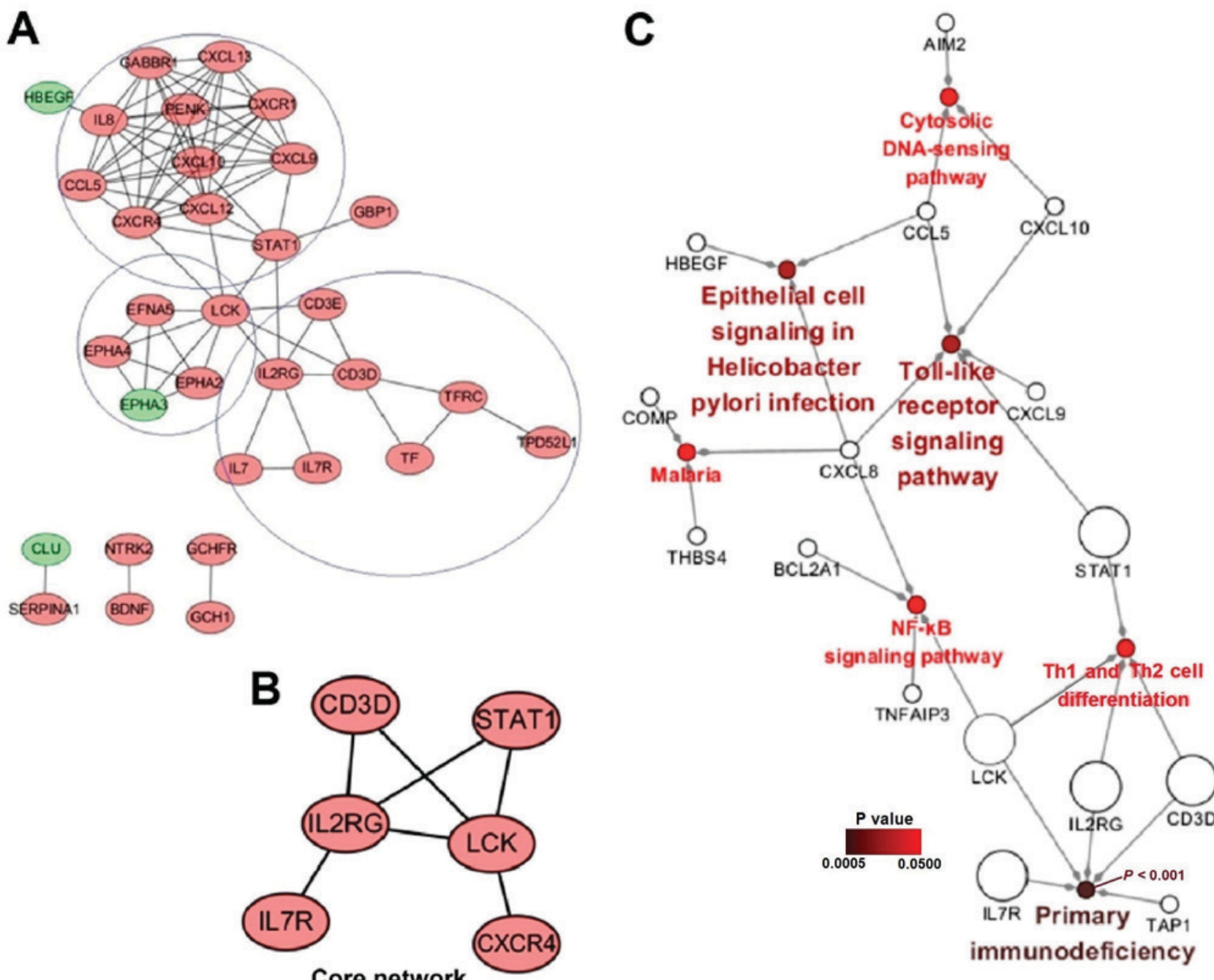

Figure 2. PPI network analysis, Core network and Gene-pathway network. (A) PPI network of differentially expressed genes (light red, upregulated; green, downregulated). (B) Core of the specific network affecting RA development. (C) Gene-pathway network associated with the development of RA. Larger circles represent genes in the core network. In Cytoscape 3.5.1 software, there are two visual styles, groups and significance. When 'significance' and 'show only pathways with P-values $<0.05$ ' were selected, the colors and the names of the enriched pathways in the figures are consistent with the P-values, therefore the colors of the pathway circles and their accompanying names represent P-values. RA, rheumatoid arthritis; PPI, protein-protein interaction.

Finally, ROC analyses suggested that the detection of $I L 7 R+$ STATl together exhibited a higher diagnostic performance compared with the detection of either IL7R or STAT1 alone (AUC=0.9464; 95\% CI: 0.8962-0.9966), with a sensitivity of $93.94 \%$ and a specificity of $80.77 \%$ (data not shown).

Validation using an additional, dependent dataset. To investigate the reliability of the results of the ROC analyses obtained from all the three datasets and to identify if there was any possible overlapping between them, the same ROC analysis, including data from the GSE36700 dataset was performed, and the results are presented in Fig. 4. Notably, it was demonstrated that the six genes in the core network exhibited good performance in distinguishing RA from OA. In addition, the AUCs of genes identified in the GSE36700 dataset were increased compared with the results obtained from the aforementioned three datasets. In conclusion, the results suggested that the results obtained from the GSE36700 dataset were closely associated with those obtained from the GSE55235, GSE55584 and
GSE55457 datasets, which further confirmed the reliability of the aforementioned results.

\section{Discussion}

RA and OA are the most common forms of degenerative joint diseases. They are the leading cause of chronic disability and may exhibit common clinical etiology $(23,24)$. However, there remains a paucity of studies investigating the sensitivity and specificity of detection indicators for identification of the two diseases, particularly for patients with advanced-stage RA or OA. Recently, epigenetic dysregulation of cartilage genes has been demonstrated to have an important role in RA and OA development (24). Despite advances in the field, biomarkers associated with the pathogenesis and progression of RA and $\mathrm{OA}$ are not well characterized. Therefore, investigation of the gene signatures associated with disease development in RA and OA may elucidate the molecular mechanisms underlying pathogenesis and identify potential therapeutic strategies for the development of a biomarker of differential diagnosis. 

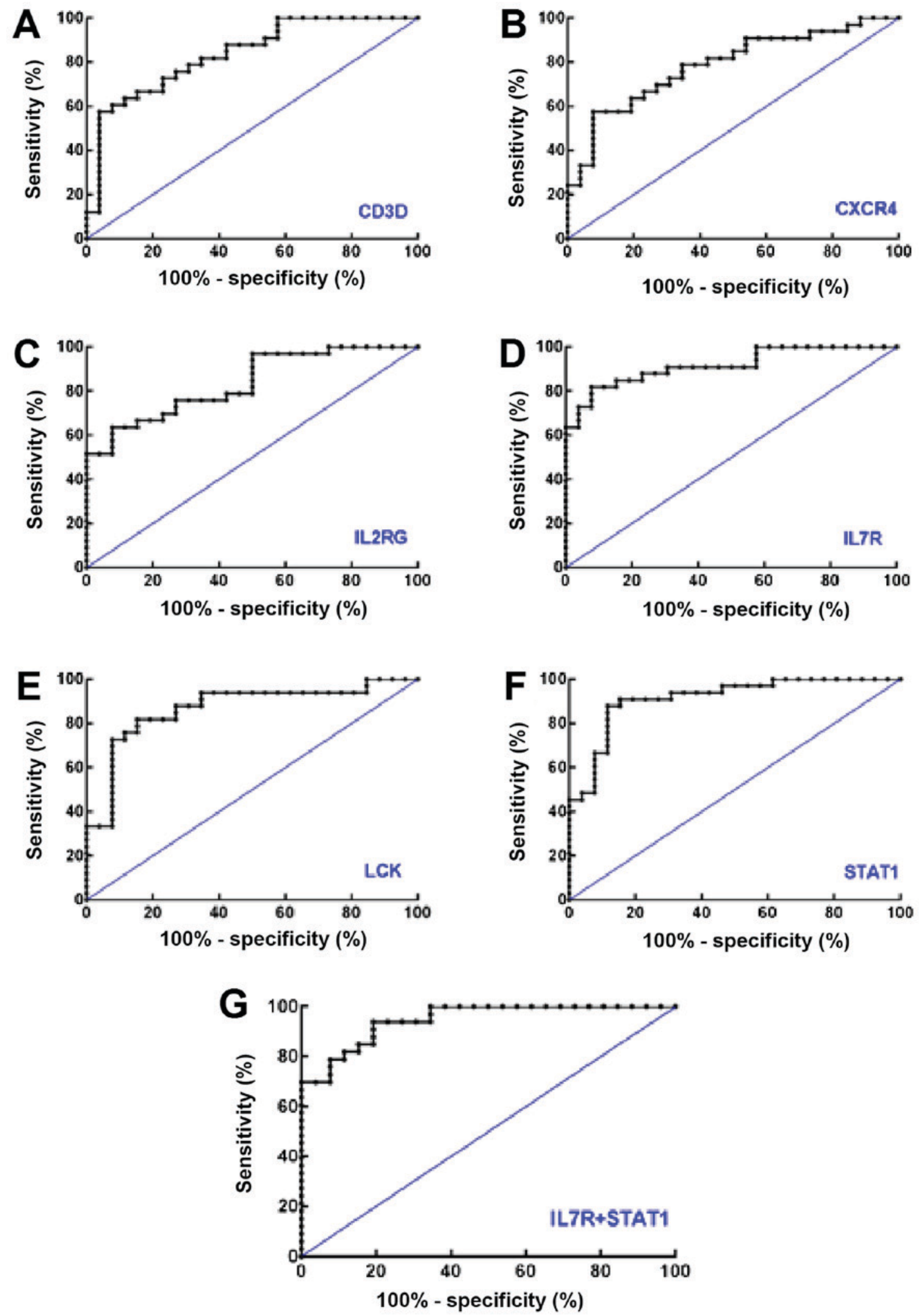

Figure 3. Receiver operating characteristic curves of the six genes in the core network to distinguish rheumatoid arthritis from osteoarthritis using data from the GSE55235, GSE55457 and GSE55584 datasets. Receiver operating characteristic curves of (A) CD3D, (B) CXCR4, (C) IL2RG, (D) IL7R, (E) LCK, (F) STAT1 and (G) IL7R+STAT1 are presented. CD3D, T-cell surface glycoprotein CD3 $\delta$ chain; CXCR4, C-X-C motif chemokine receptor 4; IL2RG, interleukin 2 receptor $\gamma$; IL7R, interleukin 7 receptor; LCK, LCK proto-oncogene, Src family tyrosine kinase; STAT1, signal transducer and activator of transcription 1.

In previous years, bioinformatics has had an increasingly important role in examining the pathogenesis of multifactorial disorders (25). In the present study, a comprehensive and systematic bioinformatics analysis of three gene expression profile datasets identified 80 significant DEGs, including 49 upregulated and 31 downregulated genes that may be 
Table II. Optimal cut-off points and associated diagnostic values of six genes in the core network as determined by receiver operator characteristic analysis.

\begin{tabular}{lccccc}
\hline Genes & Cut-off value, $\log _{2}$ & Sensitivity, $\%$ & Specificity, $\%$ & AUC & AUC 95\% CI \\
\hline CD3D & 8.65 & 66.67 & 84.62 & 0.8357 & $0.7329-0.9384$ \\
CXCR4 & 10.86 & 57.58 & 92.31 & 0.7855 & $0.6696-0.9015$ \\
IL2RG & 9.27 & 63.64 & 92.31 & 0.8368 & $0.7381-0.9356$ \\
IL7R & 8.11 & 81.82 & 92.31 & 0.9161 & $0.8466-0.9856$ \\
LCK & 6.58 & 81.82 & 84.62 & 0.8683 & $0.7718-0.9648$ \\
STAT1 & 7.36 & 90.91 & 69.23 & 0.9138 & $0.8404-0.9871$ \\
\hline
\end{tabular}

Greater AUC values indicated a greater diagnostic value. The six AUCs all demonstrated statistical significances, $\mathrm{P}<0.0001$. CD3D, T-cell surface glycoprotein CD3 $\delta$ chain; CXCR4, C-X-C motif chemokine receptor 4; IL2RG, interleukin 2 receptor $\gamma$; IL7R, interleukin 7 receptor; LCK, LCK proto-oncogene, Src family tyrosine kinase; STAT1, signal transducer and activator of transcription 1; AUC, area under the curve; CI, confidence interval.

Table III. Binary logistic regression results of the core network for rheumatoid arthritis diagnosis.

\begin{tabular}{lcccccr}
\hline Genes & $\beta$ & S.E. & Wald & OR & OR 95\% CI & P-value \\
\hline IL7R & 1.515 & 0.561 & 7.307 & 4.551 & $1.517-13.657$ & 0.007 \\
STAT1 & 1.073 & 0.503 & 4.551 & 2.923 & $1.091-7.829$ & 0.033 \\
\hline
\end{tabular}

IL7R, interleukin 7 receptor; STAT1, signal transducer and activator of transcription 1; $\beta$, coefficient of logistic regression; S.E., standard error of $\beta$ value; Wald, Wald value of Wald tests; OR, Odds ratio; OR 95\% CI, 95\% confidence interval of OR value.

associated with the development of RA and OA. These results suggested that alterations in gene expression profiles in synovial tissue may affect the development of RA and OA. Therefore, detailed analysis of the biological functions of the DEGs may be utilized to further understand the pathogenesis of the two diseases and may additionally reveal biomarkers for more accurate identification of RA and OA.

A previous study demonstrated that RA development may depend on a common alteration in the expression pattern of specific key genes (26), which was consistent with the results of the present study. Numerous previous studies have identified specific genes associated with RA development. For example, Ma et al (27) identified numerous genes (including adiponectin, ClQ and collagen domain containing, 3'-phosphoadenosine 5'-phosphosulfate synthase 1, DNA methyltransferase 1 and TIMP metallopeptidase inhibitor 1) involved in immune responses and inflammatory responses. Microarray analysis has additionally identified disease spectrum features in rheumatology and identified additional genes that may be associated with RA $(28,29)$. Biswas et al (30) identified a number of different biomarkers, genes and pathways, the majority of which have not been revealed in other studies. Differential diagnoses of RA and OA remain clinically challenging due to substantial etiological similarities (16). Microarray experiments performed by Wang et al (31) identified an overview of differences in OA gene expression compared with healthy patients and identified 85 DEGs. In conclusion, these studies suggested that RA and OA have complex pathogenic mechanisms, and future studies should perform comprehensive and systematic analyses to further elucidate these mechanisms.

Biological function and KEGG pathway enrichment analyses identified that 80 overlapped DEGs were significantly enriched in immune, inflammation, apoptosis and antioxidant stress-associated functions and pathways, including 'cytokine-cytokine receptor interaction', 'axon guidance', 'chemokine signaling pathway' and 'primary immunodeficiency'. A constructed PPI network additionally demonstrated that RA progression was associated with immunodeficiency. RA has been well established to represent a progressive, chronic, inflammatory and destructive joint disease (2). These results were based on three high throughput microarray datasets with multi-center design and containing large sample numbers of synovial tissue, which may provide further evidence for future research. In the present study, the PPI network studies demonstrated that CXCR4, LCK, IL2RG and $C D 3 D$ may represent potential biomarkers associated with immunodeficiency in RA. To confirm this inference, a more complete and specific biological network based on GeneMANIA was determined, from which a core network of 293 candidate genes that may affect RA and OA development was obtained. Furthermore, the fact that the core network was closely aligned with the constructed PPI network further suggested that the six genes in the core network are involved in the occurrence and development of RA and OA.

To further investigate how these genes exhibit their biological function and affect the occurrence of RA, a gene-pathway interaction network was constructed. The 

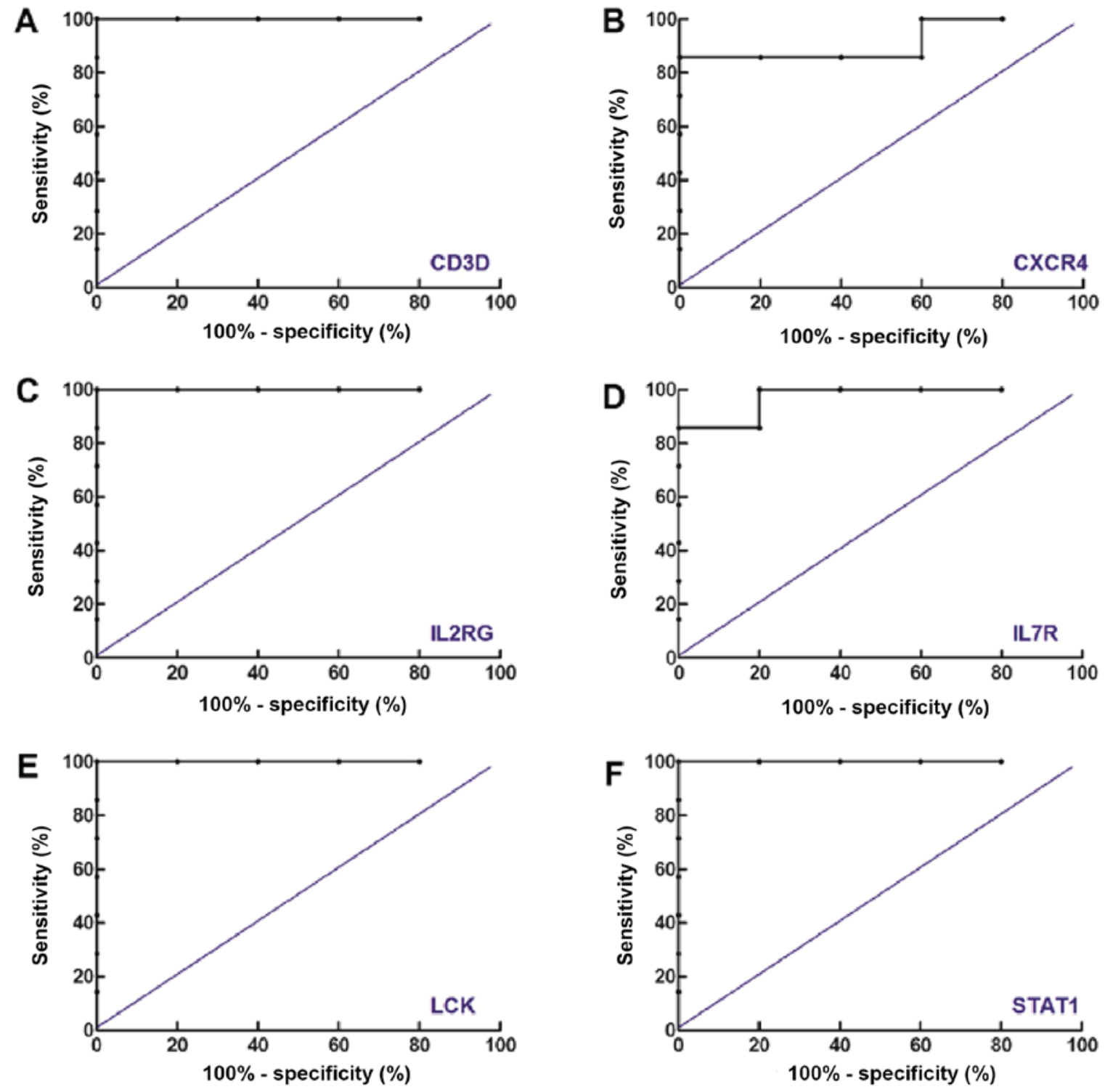

Figure 4. Receiver operating characteristic curves of the six genes in the core network to investigate the differentiation between rheumatoid arthritis and osteoarthritis using data from the GSE36700 dataset. Receiver operating characteristic curves of (A) CD3D, (B) CXCR4, (C) IL2RG, (D) IL7R, (E) LCK and (F) STAT1 are presented. CD3D, T-cell surface glycoprotein CD3 $\delta$ chain; CXCR4, C-X-C motif chemokine receptor 4; IL2RG, interleukin 2 receptor $\gamma$; IL7R, interleukin 7 receptor; LCK, LCK proto-oncogene, Src family tyrosine kinase; STAT1, signal transducer and activator of transcription 1.

results demonstrated that in the gene-pathway interaction network, five genes in the core network $(C D 3 D, I L 2 R G, I L 7 R$, $L C K$ and $S T A T 1$ ) were included and notably, these genes were demonstrated to interact with the primary immunodeficiency pathway either directly (CD3D, IL2RG, IL7R and $L C K)$ or indirectly (STAT1). Therefore, the results suggested that altered expression levels of $C D 3 D, I L 2 R G, I L 7 R, L C K$ and $S T A T 1$ may activate the primary immunodeficiency pathway and subsequently lead to primary immune system dysfunction and the development of RA.

Primary immunodeficiencies are a heterogeneous group of disorders that cause increased susceptibility to infection, autoimmune disease and malignancy (32). From the primary immunodeficiency pathway, IL7R, LCK and Janus kinase (JAK)3/STAT1 primarily affect T-cell differentiation and antibody production (33), which may represent the basis of RA development. Investigation of the diagnostic capacity to distinguish RA from OA suggested that the genes in the core network may be detected alone to predict and diagnose RA occurrence with high sensitivity and specificity; however, the combined detection of important indicators may improve the effectiveness of this diagnostic strategy. Therefore, binary logistic regression analysis was used to screen for $I L 7 R$ and STAT1 simultaneously to improve RA diagnosis.

A previous study identified that STAT1 is important in RA occurrence and is upregulated in patients with RA (34), which corroborates the results of the present study. STAT1 has been widely regarded to represent an important transcription factor involved in joint inflammation and destruction $(33,35)$. STAT1 may be activated by numerous cytokines that are expressed in RA synovium, including interferon (IFN) $\gamma$, type I IFNs, IL6, IL10 and IL27, which induce inflammation via direct or indirect activation of mitogen-activated protein kinase, JAK-STAT and nuclear factor- $\kappa \mathrm{B}$ signaling pathways (36). 
The functional defects in important proteins (including IL7R and IL7) associated with the IL7 signaling pathway may be involved in the pathogenesis of severe combined immunodeficiency (SCID) (36). IL7R was identified as a novel molecule with a potential role in RA in the present study. IL7R has been identified to have a critical role in $\mathrm{V}(\mathrm{D}) \mathrm{J}$ recombination during lymphocyte development, and thus mutations in this gene may increase the risk of SCID (37).

The identification of these two key biomarkers and a key pathway associated with immunodeficiency in the development of RA and reveals novel therapeutic targets for anti-immunotherapy for patients with RA.

In conclusion, the present study demonstrated that STAT1 and the primary immunodeficiency pathway may be precisely utilized to differentiate RA from OA. In addition, the present study additionally identified a previously unreported novel biomarker (IL7R), which may serve as potential candidate biomarker to differentiate RA from OA at the time of diagnosis. Therefore, the present study demonstrated potential implications for future clinical management of patients with RA and OA.

\section{Acknowledgements}

The authors would like to thank Dr Zhenzhong Li from Beijing Compass Biotechnology Co., Ltd. (Beijing, China) for his help with part of the data analysis.

\section{Funding}

The present study was supported by grants from the National Natural Science Foundation of China (grant nos. 81773372 and 81573104).

\section{Availability of data and materials}

The datasets used and/or analyzed during the current study are available from the National Center for Biotechnology Information's Gene Expression Omnibus database (http://www. ncbi.nlm.nih.gov/geo/).

\section{Authors' contributions}

YX and RZ designed the study. JW, LH, XY, AY, JZ, BL, DZ and ZL acquired the data. RZ, YX and JW analyzed and interpreted the data, and drafted the manuscript. All authors critically revised the manuscript, and read and approved the final version of the manuscript.

\section{Ethics approval and consent to participate}

Not applicable.

\section{Patient consent for publication}

Not applicable.

\section{Competing interests}

The authors declare that they have no competing interests.

\section{References}

1. García-Bermúdez M, López-Mejías R, González-Juanatey C, CastañedaS,Miranda-FilloyJA,BlancoR,Fernández-GutiérrezB, Balsa A, González-Alvaro I, Gómez-Vaquero C, et al: Lack of association between TLR4 rs4986790 polymorphism and risk of cardiovascular disease in patients with rheumatoid arthritis. DNA Cell Biol 31: 1214-1220, 2012.

2. Song YJ, Li G, He JH, Guo Y and Yang L: Bioinformatics-based identification of MicroRNA-regulated and rheumatoid arthritis-associated genes. PLoS One 10: e0137551, 2015.

3. Liu G, Jiang Y, Chen X, Zhang R, Ma G, Feng R, Zhang L, Liao M, Miao Y, Chen Z, et al: Measles contributes to rheumatoid arthritis: Evidence from pathway and network analyses of genome-wide association studies. PLoS One 8: e75951, 2013.

4. Ballard DH, Aporntewan C, Lee JY, Lee JS, Wu Z and Zhao H: A pathway analysis applied to genetic analysis workshop 16 genome-wide rheumatoid arthritis data. BMC Proc 3 (Suppl 7): S91, 2009.

5. Wang H, Guo J, Jiang J, Wu W, Chang X, Zhou H, Li Z and Zhao J: New genes associated with rheumatoid arthritis identified by gene expression profiling. Int J Immunogenet 44: 107-113, 2017.

6. Mc Ardle A, Flatley B, Pennington SR and FitzGerald O: Early biomarkers of joint damage in rheumatoid and psoriatic arthritis. Arthritis Res Ther 17: 141, 2015.

7. Carlson A, Bothner B and June R: Toward OA biomarkers: Metabolomic profiles of synovial fluid from OA, RA, and healthy patients. Osteoarthritis Cartilage 25 (Suppl 1): S94-S95, 2017.

8. Atif U, Philip A, Aponte J, Woldu EM, Brady S, Kraus VB, Jordan JM, Doherty M, Wilson AG, Moskowitz RW, et al: Absence of association of asporin polymorphisms and osteoarthritis susceptibility in US Caucasians. Osteoarthritis Cartilage 16: 1174-1177, 2008.

9. Bijsterbosch J, Kloppenburg M, Reijnierse M, Rosendaal FR, Huizinga TW, Slagboom PE and Meulenbelt I: Association study of candidate genes for the progression of hand osteoarthritis. Osteoarthritis Cartilage 21: 565-569, 2013.

10. Fang H, Zhang F, Li F, Shi H, Ma L, Du M, You Y, Qiu R, Nie H, Shen L, et al: Mitochondrial DNA haplogroups modify the risk of osteoarthritis by altering mitochondrial function and intracellular mitochondrial signals. Biochim Biophys Acta 1862: 829-836, 2015.

11. Zhang M, Mu H, Lv H, Duan L, Shang Z, Li J, Jiang Y and Zhang R: Integrative analysis of genome-wide association studies and gene expression analysis identifies pathways associated with rheumatoid arthritis. Oncotarget 7: 8580-8589, 2016.

12. González-Huerta NC, Borgonio-Cuadra VM, Zenteno JC Cortés-González S, Duarte-Salazar C and Miranda-Duarte A: D14 repeat polymorphism of the asporin gene is associated with primary osteoarthritis of the knee in a Mexican Mestizo population. Int J Rheum Dis 20: 1935-1941, 2017.

13. Young SP, Kapoor SR, Viant MR, Byrne JJ, Filer A, Buckley CD, Kitas GD and Raza K: The impact of inflammation on metabolomic profiles in patients with arthritis. Arthritis Rheum 65: 2015-2023, 2013.

14. Arnett FC, Edworthy SM, Bloch DA, McShane DJ, Fries JF, Cooper NS, Healey LA, Kaplan SR, Liang MH, Luthra HS, et al: The American rheumatism association 1987 revised criteria for the classification of rheumatoid arthritis. Arthritis Rheum 31: 315-324, 1988.

15. Altman R, Asch E, Bloch D, Bole G, Borenstein D, Brandt K, Christy W, Cooke TD, Greenwald R, Hochberg M, et al: Development of criteria for the classification and reporting of osteoarthritis. Classification of osteoarthritis of the knee. Diagnostic and therapeutic criteria committee of the American rheumatism association. Arthritis Rheum 29: 1039-1049, 1986.

16. Woetzel D, Huber R, Kupfer P, Pohlers D, Pfaff M, Driesch D, Häupl T, Koczan D, Stiehl P, Guthke R and Kinne RW: Identification of rheumatoid arthritis and osteoarthritis patients by transcriptome-based rule set generation. Arthritis Res Ther 16: R84, 2014.

17. Nzeusseu Toukap A, Galant C, Theate I, Maudoux AL, Lories RJ, Houssiau FA and Lauwerys BR: Identification of distinct gene expression profiles in the synovium of patients with systemic lupus erythematosus. Arthritis Rheum 56: 1579-1588, 2007.

18. Green GH and Diggle PJ: On the operational characteristics of the Benjamini and Hochberg false discovery rate procedure. Stat Appl Genet Mol Biol 6: Article27, 2007. 
19. Bader GD and Hogue CW: An automated method for finding molecular complexes in large protein interaction networks. BMC Bioinformatics 4: 2, 2003.

20. Li M, Li D, Tang Y, Wu F and Wang J: CytoCluster: A cytoscape plugin for cluster analysis and visualization of biological networks. Int J Mol Sci 18: pii: E1880, 2017.

21. Montojo J, Zuberi K, Rodriguez H, Kazi F, Wright G, Donaldson SL, Morris Q and Bader GD: GeneMANIA Cytoscape plugin: Fast gene function predictions on the desktop. Bioinformatics 26: 2927-2928, 2010.

22. Youden WJ: Index for rating diagnostic tests. Cancer 3: 32-35, 1950.

23. Bay-Jensen AC, Bihlet A, Byrjalsen I, Andersen J, He Y, Siebuhr A, Thudium C, Guehring H, Michaelis M, Ladel C, et al: Elevated levels of CRPM, an inflammatory biomarker correlating with disease activity in RA, are prognostic of radiographic knee OA. Osteoarthritis Cartilage 25 (Suppl 1): S32, 2017.

24. Castrejon I, Chua JR, Malfait AM, Block JA and Pincus T: Disease burden in rheumatoid arthritis (RA) patients who have secondary osteoarthritis (OA) is lower than in primary $\mathrm{OA}$ but higher than in RA with no secondary OA. Osteoarthritis Cartilage 25: S218-S219, 2017.

25. Can T: Introduction to bioinformatics. Methods Mol Biol 1107: $51-71,2014$

26. Huber R, Hummert C, Gausmann U,Pohlers D, Koczan D, Guthke R and Kinne RW: Identification of intra-group, inter-individual, and gene-specific variances in mRNA expression profiles in the rheumatoid arthritis synovial membrane. Arthritis Res Ther 10: R98, 2008.

27. Ma C, Lv Q, Teng S, Yu Y, Niu K and Yi C: Identifying key genes in rheumatoid arthritis by weighted gene co-expression network analysis. Int J Rheum Dis 20: 971-979, 2017.

28. Li G, Han N, Li Z and Lu Q: Identification of transcription regulatory relationships in rheumatoid arthritis and osteoarthritis. Clin Rheumatol 32: 609-615, 2013.

29. Yi CQ, Ma CH, Xie ZP, Cao Y, Zhang GQ, Zhou XK and Liu ZQ Comparative genome-wide gene expression analysis of rheumatoid arthritis and osteoarthritis. Genet Mol Res 12: 3136-3145, 2013.
30. Biswas S, Manikandan J and Pushparaj PN: Decoding the differential biomarkers of Rheumatoid arthritis and Osteoarthritis: A functional genomics paradigm to design disease specific therapeutics. Bioinformation 6: 153-157, 2011.

31. Wang $X$, Ning $Y$ and Guo $X$ : Integrative meta-analysis of differentially expressed genes in osteoarthritis using microarray technology. Mol Med Rep 12: 3439-3445, 2015.

32. Nayan S, Alizadehfar R and Desrosiers M: Humoral primary immunodeficiencies in chronic rhinosinusitis. Curr Allergy Asthma Rep 15: 46, 2015.

33. Paciolla M, Pescatore A, Conte MI, Esposito E, Incoronato M, Lioi MB, Fusco F and Ursini MV: Rare mendelian primary immunodeficiency diseases associated with impaired $N F-\kappa B$ signaling. Genes Immun 16: 239-246, 2015.

34. Jiang LJ, Zhang NN, Ding F, Li XY, Chen L, Zhang HX, Zhang W, Chen SJ, Wang ZG, Li JM, et al: RA-inducible gene-I induction augments STAT1 activation to inhibit leukemia cell proliferation. Proc Natl Acad Sci USA 108: 1897-1902, 2011.

35. Lim JY, Im KI, Lee ES, Kim N, Nam YS, Jeon YW and Cho SG: Enhanced immunoregulation of mesenchymal stem cells by IL-10-producing type 1 regulatory $\mathrm{T}$ cells in collagen-induced arthritis. Sci Rep 6: 26851, 2016.

36. Yokota A, Narazaki M, Shima Y, Murata N, Tanaka T, Suemura M, Yoshizaki K, Fujiwara H, Tsuyuguchi I and Kishimoto T: Preferential and persistent activation of the STAT1 pathway in rheumatoid synovial fluid cells. J Rheumatol 28: 1952-1959, 2001.

37. Pongratz G, Anthofer JM, Melzer M, Anders S, Grässel S and Straub RH: IL-7 receptor $\alpha$ expressing B cells act proinflammatory in collagen-induced arthritis and are inhibited by sympathetic neurotransmitters. Ann Rheum Dis 73: 306-312, 2014.

This work is licensed under a Creative Commons Attribution-NonCommercial-NoDerivatives 4.0 International (CC BY-NC-ND 4.0) License. 